\title{
Introduction
}

\section{Causes and Functions of Linguistic Awareness in Language Acquisition: Some Introductory Remarks}

\author{
W.J.M. Levelt, A. Sinclair, and R.J. Jarvella \\ Max-Planck-Gesellschaft, Projektgruppe für Psycholinguistik \\ Berg en Dalseweg 79, Nijmegen, The Netherlands
}

In order to introduce this book to the reader, we will give a short outline sketch of the main theoretical and empirical questions that we feel must be discussed if one endeavors to study the growth of linguistic awareness in the child. This sketch will at the same time be used to introduce the different papers in this book, seen in the light of the general framework which emerges. This restriction in viewpoint should be kept in mind. The introduction is not meant to be a review of the contributions to the present volume, and will not do justice to all (nor perhaps even the greater part) of the issues taken up in the various papers. It will be no more than an independent introductory discussion of the field, in which special reference is made to the work printed here.

The way we will proceed is as follows. Firstly some examples of the kind of data and phenomena with which we are concerned will be presented. After this purely descriptive section, the main theoretical issues as we see them will be discussed in an abstract and rather summary fashion. Few details will be given, since many of these issues are more extensively discussed in different places in the book. Rather, we will try to provide a peg for connecting some of the theoretical questions raised in the different papers. This will be done by concentrating on three central issues: the epistemological status of linguistic awareness, its causation, and its possible functions in language acquisition. 


\section{DATA AND PHENOMENA}

There are different ways of classifying phenomena of linguistic awareness observable in the child. Ultimately, the classification arrived at will depend on one's theory of structure and function in the child's growing conception of language. But on a preliminary, atheoretical level, there are two obvious ways of structuring the phenomena in question. The first of these is to use a criterion of explicitness. Some metalinguistic phenomena are at the border of awareness, whereas others are clearly the result of very explicit reflection on language. Examples of the first extreme are for instance self-corrections, which occur frequently in normal speech, and which are readily observable in child speech as well. Re-starts can show that the child was aware that what he began or was about to say was inappropriate or incorrect. These phenomena of awareness are very ethereal, going mostly unnoticed by the listener. For the child speaker they may also pass by on the stream of consciousness without leaving a trace.

Explicit reflections on language can likewise be observed in children. One passage from Gleitman, Gleitman, and Shipley (1972) suffices to show this:

Adult: How about this: Claire Zoves Claire.

Child (7): Claire loves herself sounds much better.

Adult: Would you ever say Claire loves Claire?

Child: Well, if there is somebody Claire knows named Claire. I know somebody named Claire, and maybe I'm named Claire. etc. (p. 150)

The ultimate form of explicitness is what linguists regale us with when they formulate their intuitions concerning the structure or grammaticality of sentences in the form of rules; and in every child there is a budding linguist.

Between the two extremes one can find various degrees or levels of explicitness. Children not only correct themselves, but also others. This requires not only awareness of linguistic trouble but at the same time some ability to formulate what went wrong. Children spontaneously play with language (cf. Weir, 1962), just as they play with other things. In these games language becomes divorced from conventional use--it is no longer a means of communication, but an object of conscious activity. Games involving rhyme and word substitution can be observed quite early in children (Kaper, 1959). Read's chapter in this volume reports, among other things, on ex- 
periments where children are induced to choose words that rhyme with other words. The results provide substantial insight into the children's conception of the English vowel system. Even more explicit manipulation of language can be observed when the child becomes familiar with the printed word. Again, Read's paper gives abundant information about the increasing analytic and syntactic capacities which accompany the acquisition of written language. Lundberg, in his contribution, gives various experimental indications about how these faculties develop. Several linguistic notions in the adult language user seem to derive from early stages of explicit phonological and morphological analysis through which the child has to pass to acquire reading and writing. This seems to be specially true for the notion of "word" itself, to which we will return. The most explicit expressions of awareness are obtained by direct questioning, as in the example given above. One can ask the child about grammaticality, structure, cohesion of sentence parts, case roles (see Braine \& Wells, 1978), or appropriateness of speech acts (see especially Robinson \& Robinson, 1977), and the older the child the more detailed are the answers one receives. Examples of such explicit questionanswering can be found at various places in this book. Flores d'Arcais asked children to judge the similarity of meaning between various connectives and between sentences containing the connectives. Question-answering about grammaticality is reported in Slobin's paper. And Hirsch, Gleitman, and Gleitman interrogated children about ambiguity.

This is not the place to give a full review of the levels of explicitness, and types of phenomena that correspond to them. That is done in a detailed fashion in E. Clark's review paper in this book. Rather we would like to juxtapose this classification scheme with a second one, which is simply the criterion of developmental stage. One may ask which forms of linguistic awareness are first to appear, and which come later. Among the first phenomena one can observe is the child coping with the failures of speech acts. If the one-and-a-half year old child addresses its mother verbally, and the mother pretends not to understand, by saying mhm? for example, the child is likely to restate the utterance with minor variations, and this may be repeated several times (see for instance, Foppa 1978, and the work of Käsermann referred to there). The variations are probably not random (cf. Marshal1 \& Morton's paper), and seem to indicate that the child is aware that its utterance is in need of some kind of change or improvement. Spontaneous self-corrections, i.e., ones not instigated by an adult, occur almost as 
early. These initial expressions of linguistic awareness all seem to serve the function of helping establish effective commication, but it is not much later that verbal play appears; and this seems to imply awareness of a very different form. Verbal play may involve almost any aspect of language, witness Weir's (1962) surprising observations, and does not serve any immediate communicative function.

It is not much before the age of five or six that the child begins to draw explicit distinctions between the form and meaning of a word or utterance (Papandropoulou \& H. Sinclair, 1974, and especially Papandropoulou's contribution to the present volume), and it is not yet known when meanings and functions of utterances become distinguished as occurs in indirect speech acts. At about the same age, the child develops sensitivity to puns and ambiguity--the separation of form and meaning permitting him to understand and make verbal jokes--and this is evidently an enduring source of enjoyment at school age. This sensitivity is the main topic of the chapter by Hirsch, Gleitman, and Gleitman. In a controlled experiment, they show that awareness of ambiguity develops relatively late in childhood.

The acquisition of reading and writing seems to prefigure the adult ability to explicitly discuss the form and function of language. Not only are the analytic capacities of the child enormously sharpened by the acquisition of spelling and writing, but the visual mode dispels with one of the major problems the child has previously had to cope with in reflecting on his own language: a written word or text remains present and can be studied repeatedly; it need not be held in memory to be reflected upon. The chapters by Read and Lundberg detail some of these developments. In addition, however, formal teaching in school provides children with an extensive metalinguistic vocabulary. Not only does the meaning of the word word come into full reach of the child, but so do various technical linguistic terms such as syzzable, noun, verb, subject, object, etc. On the other hand, the development of a metalinguistic vocabulary doesn't require explicit teaching. Slobin, in his paper, describes the early metalinguistic vocabulary of a bilingual child, and Heeschen's article describes a rich metalinguistic vocabulary of an illiterate society still in the stone age.

There is an unmistakable connection between the criteria of developmental stage and explicitness: the older the child, the greater his facility to reflect upon language. It should, however, be stressed that different levels of explicitness are simultaneously observable at all stages of development. 
Self-corrections, for instance, are not only very early expressions of linguistic awareness, but very permanent ones as well: they can be observed in adult language usage and the young child's speech equally well. The same can be said about verbal play, and many other expressions of linguistic awareness. The most comprehensive treatment of relevant data and phenomena in this volume can be found in E. Clark, whose contribution pulls together most of the evidence on linguistic awareness scattered throughout the literature on child language.

\section{THEORETICAL ISSUES}

Though the first priority on entering this still largely unexplored area is to obtain descriptive evidence about the child's growing linguistic awareness, as much theoretical framework as possible should be kept in mind while gathering it. It seems to us that three major theoretical issues should be distinguished. These concern: (1) the epistemological status of linguistic awareness; (2) the causes of linguistic awareness and insight; and (3) the functions of linguistic awareness in development and language use. Let us shortiy review these issues in turn.

\section{Epistemological Status of Linguistic Awareness}

What does it mean to be aware of a linguistic state of affairs? Even if one acknowledges that phenomena of linguistic awareness vary greatly, not only with respect to explicitness, but also with respect to content, this very general preliminary question must nevertheless be asked. One answer, which has been rather dominant in the linguistic literature, is that awareness is implicit knowledge that has become explicit. Clearly, this notion goes back to Chomsky's theory of linguistic competence. Competence is tacit knowledge of the language--it exists in the form of linguistic intuitions, which can sometimes be made explicit through questioning or by means of other procedures, and may take the outward form of linguistic judgments (concerning the acceptability of utterances, etc.). Extensive discussion of the epistemological status of this theory can be found in Levelt $(1972,1974)$, and there is no need to repeat it here. The main conclusions, however, are first that if there is such a thing as a unified competence underlying a 11 linguistic behavior, then explicit intuitions have at most a highly indirect and involved relation to this base of tacit knowledge. Contrary to what has generally been claimed, the relations between explicit intuitions and underlying 
competence are less direct than those between phenomena of primary language usage (speaking, listening) and competence. Second, linguistic judgment is a form of behavior which should be explained in its own right, just like any other form of linguistic behavior. It has no special epistemologica status. This is, more generally, true for linguistic awareness: it is a phenomenon to be explained. There is no reason whatsoever to assume that it has a special "hot line" to implicit knowledge. This point is taken up further in Seuren's contribution to this book. That phenomena of linguistic awareness have no privileged status, and require explanation just as other linguistic phenomena do, is again strongly argued by Read, and by Hirsch et al. in this volume.

These statements should not be read as implying that linguistic awareness is a "mere" epiphenomenon which accompanies certain unconscious 1 inguistic processes but never play a functional role in them itself. At this point one should make an important epistemological distinction. If one is aware of a linguistic entity $X, X$ might be one of the causes of this awareness. For instance, $X$ could be built-in property of the language-producing mechanism, and be part of the cause of a speaker expressing some form of awareness of $x$. On the other hand, $x$ could be nothing else than the intentional object of the user's awareness, without having any real existence outside this intention. This is what could be called the "epiphenomenon" situation. Finally, $X$ could be both cause and intentional object: the thing one is aware of does have a real existence, and (partiy) caused awareness to come about. This final view is very much in line with a realist interpretation of perception. (The real existence of object 0 in part causes the perception of 0.) A similar realist interpretation of "being aware of linguistic entity $X "$ seems rather strong, and there is reason to be careful not to jump too rapidly from an intentional object interpretation of linguistic awareness to a cause interpretation.

Perhaps the mechanism proposed in Marshall and Morton's paper comes closest to what we have in mind here. We will return to this matter in the next paragraphs. For the moment, let us simply stress that neither the adult, nor the child, can become aware of the biological or mental machinery involved in language use. In history a similar point has been made over and over again with respect to other cognitive abilities. It is what Bühler (1907, 1908) claimed for conscious phenomena in thinking, Piaget (1974a, 1974b) for complex motoric and cognitive performance, and Nisbett and Decamp Wilson (1977) for a large variety of cognitive processes. H. Sinclair in this volume 
discusses the Piagetian point of view. She explains that "becoming aware" is a mental activity which interacts with other cognitive processes. The subject is almost fully unconscious of the internal mechanisms of cognitive functioning and of those involved in "becoming aware." Seuren, in his paper, makes the same point for linguistic functioning. Nisbett and Decamp Wilson's main point is that there is very little evidence in the literature that people are conscious of many of their own mental processes. Awareness seems to be restricted to the outcome or results of such processes, and if people do report on processes, this is--they contend--usualiy a logical reconstruction of how such a result might have come about (often in the form of a motivation) rather than a memory trace of the process itself. It should be added, however, that the distinction between process and outcome is not so obvious as it may seem at first sight. If knowledge is procedural, as is often claimed in the artificial intelligence literature, then results of procedures are procedures again, and the distinction made by Nisbett and Decamp Wilson fades considerably.

It is especially in the case of "slow" mental processes, such as thinking in chess (De Groot, 1965), that subjects seem to be able to describe the process more or less on 1 ine. In this way protocol analysis contributed quite essentially to our knowledge of problem solving (Newell \& Simon, 1972). Whether this is in agreement with Nisbett and Decamp Wilson, or Buihler for that matter, is an issue in itself; what is relevant here is that, as opposed to problem solvers, language users show practically no insight into the way they perceive or produce language. Protocol analysis is simply not a feasible technique for analyzing language understanding or production. What is involved is, as a rule, very much in the dark for the language user himself. It is thus all the more interesting to investigate under what conditions the language user does become aware of his speaking or listening processes.

Read, in his article, shows that linguistic awareness can be provoked in children, or more precisely that by questioning a child about his language, the child acquires access to linguistic structure of which he showed no spontaneous awareness beforehand. It is indeed important to make a distinction between spontaneous manifestations of awareness (in daily usage), and potential accessibility of 1 inguistic processes and structures. There is no doubt that the latter has definite limits, but these limits and how they retreat as the child develops deserve careful examination. 
Causes of Linguistic Awareness

Skills like driving a car can become almost completely automatic with increasing experience. By "automatic," we mean that the activity requires little conscious attention. For example, while at the wheel an experienced driver can simultaneously and attentively discuss a complicated issue with his fellow passenger. Skills are, by definition, automated activities, in which low-level decisions (such as steering or accelerating) do not require conscious attention. Attention can be reserved for more general, high-level reflection and decisions (such as where to go, and when to get the tank filled). In performing complex skills, moreover, low-level decisions often have to be taken far too rapidiy to be handled by conscious decision-making. If they are taken consciously, the activity may become disturbed and break down: indeed, this is what we observe in beginning drivers, who do have to pay attention to nearly all the elements in the total action pattern. Linguistic skills are no different in this respect (see Levelt \& Kempen, 1978; Levelt, 1978). In speaking, for instance, low-level decisions-including, for example, choice of syntactic frames and articulatory activities-apparently require no, or almost no, conscious planning decisions. Attention is usually directed to the content of what is going to be expressed, not to the language used for it. The latter develops in a largely automated fashion.

For any skill, however, there are two obvious circumstances where conscious reflection or decision-making, and therefore awareness, occur. The first one is during skizl acquisition, as in the example of learning to drive. Automatization of elementary activities is often preceded by a stage of conscious learning of these activities. Whether a conscious stage also precedes automatization of elementary speech activities during language acquisition is still an open question. We will return to this point in the next section.

The second circumstance where awareness can be observed in the execution of skills is at moments of failure, i.e., when unexpected or undesired results occur. If suddenly the car one is driving starts to skid, one becomes fully aware of what is happening, and conscious decision-making may result (if there is sufficient time left). A similar claim can be found in Piaget (1974a) where the child becomes aware in cases of disequilibrium, i.e., where the automatic regulations in performing intentional acts are no longer sufficient to attain a goal. It is this mechanism of matching aim and result which $H$. Sinclair in her chapter applies to speech acts. In the same way, a major cause for linguistic awareness could be failure in communication, that is, 
in speaking or understanding. Repairs made while speaking, or the registering of what? while listening, may occur when automatic processing fails to yield the result being sought: a speech error is corrected or there is an attempt to remedy a lapse in understanding. Conscious intervention is then required, and the language user is--momentarily at least--in some fashion aware of the linguistic entity that caused the problem. Problems of this type abound for the young child, of course, precisely because his linguistic skills are still in a very preliminary stage of development. (For a case study of linguistic awareness in a child exposed to several languages, see Slobin's chapter.) In fact, several papers in this book show how essential this notion of failure is for the explanation of early linguistic awareness (cf. especially the chapters by E. Clark, H. Sinclair, and Marshall \& Morton). There is no need to elaborate this point here, and we turn to a set of causes which may be involved with more explicit forms of linguistic awareness.

Explicit reflections on language can be obtained from the child (as well as from the adult) by asking explicit questions, such as can one say X? or Is $X$ good English? How subjects actually manage to answer such questions is largely unknown, but one plausible procedure would be for the language user to try to think of a situation in which $x$ can be said. If it is easy to imagine such a situation, the answer may well be Yes, if it is too difficult to think one up, it may be No. Levelt et al. (1977) tested this theory by giving subjects verbal material of borderline grammaticality which related to situations that were either easy or difficult to imagine. It was indeed the case that the former material was more frequently judged to be grammatical than the latter. Subjects also answered more rapidily for material which was easy to imagine. Nevertheless, results showed that it was unlikely that the subjects, in conceiving their answers, had to find a "full" interpretation for the verbal item presented. This was concluded from a comparison with reaction times for a paraphrase task using the same material, which had a very different pattern. It is argued in that article that intuitions concerning acceptability may result from rather shallow semantic processing; this is in Iine with the findings of Mistler-Lachman (1972) on intuitions concerning meaningfulness. In the next section we will return to the possible functions of such shallow processing. Answering questions about acceptability and paraphrase (as in Flores d'Arcais' paper), about morphological structure (as in Read's paper), about word length (as in Papandropoulou's and Lundberg's contributions), and so forth, requires what Lundberg calls an "attention shift," 
i.e., a shift from content to form. Lundberg shows that the difficulty of making this shift adheres to general Gestalt principles. The stronger the semantic "Gestalt," the harder to break it down.

These few remarks about causation are admittedly vague and of a general nature; for these reasons, their value is clearly limited. The origins of linguistic awareness may in reality be as varied as the phenomena themselves. What induces the child to play linguistically, either alone in self-talk or with others by joking, to play rhyming games, and the like? These questions are far from being answered, but it might nevertheless be worthwhile to ask an even more abstract question: Is the genesis of linguistic awareness universal? And if so, which forms of awareness are universal, and which are language or culture specific? A major distinction needs to be made here between literate and $i l l i t e r a t e$ societies since learning to read and write fosters the development of linguistic notions and reflections that might otherwise not easily occur. The notion of "word," let alone notions such as "syllable," "sentence," and other general illustrations of phonological or syntactic insight, may be very writing-dependent.

Thus, Heeschen in this volume shows that the metalinguistic vocabulary of the Eipo speech community, a neolithic mountain people of New Guinea, is bound by content and appropriateness, and shows little concern for language structure proper. Like other groups, the Eipo are sensitive to various dialect differences with and between their own and neighboring cultures; their linguistic beliefs and taboos require that words be "chosen" and "used" with care. Even so, the conclusion must be guarded, since Heeschen also found that there was high agreement on fixed word and clause orders among the speakers of the language he studied. If, on the other hand, the "failure causes awareness" theory is correct, then self-corrections and other similar phenomena should be observable in all societies, since "skillfulness" is a property of all normal language use. It is much less trivial to predict whether rhyming, or for that matter intuitions of acceptability, are universal. There is a major task here for linguistic anthropologists.

A final remark should be made about asking the child metalinguistic questions, such as why-questions, that require whole explanations as answers. 0lder children can handle such questions better than younger ones, but it is probably false to ascribe this fact solely to a greater reflexive awareness of language. Explaining is a complex verbal activity; the child has to express more or less complex ideas or facts in a proper linguistic form. 
This skill is involved in explaining the way or in telling narratives, as well as in giving metalinguistic answers. The development of the child's capacity to explain verbally shouldn't be confused with his growing capacity to reflect on language: methodological care is required to keep these issues apart.

The Functions of Linguistic Awareness

For what use do we develop linguistic awareness? As Heeschen points out, awareness occurs when there is contact between languages, and historical$1 y$, rather than being exceptional, such contact is the rule. We also know that a certain sophistication in consciously manipulating language is a requirement for learning to read and write (see especially Read, this volume). Still, it seems unlikely that evolution has endowed us with awareness of language for just these purposes. Such skills seem fairly tangential even to basic language use. The more fundamental functions of linguistic awareness should probably be sought for in the facilitative role it plays in faceto-face communication, and in learning to communicate. Let us consider these in turn.

Linguistic awareness can facilitate communication. In the preceding section it was suggested that awareness can be provoked by failure. It may arise momentarily when a conversation goes awry. Conscious repair may be an effective means of coping with such moments of difficulty and helping prevent a deeper breakdown in communication. This situation is not different from the one for other complex skills. At a moment of failure, i.e., where the automated procedures produce an undesired result, conscious correction can often prevent more serious, subsequent failures. What is especially interesting about conscious linguistic repairs is that they themselves have a rather complex but systematic structure. Schegloff et al. (1977), in a recent paper, describe such regularities, and remark that "An adequate theory of the organization of natural language will need to depict how a natural language handles its intrinsic troubles" (p. 381). Language is a skill which, through its sheer complexity, is very failure-prone, and it has developed its own mechanisms of coping with this, among them conscious repairing. E. Clark's paper in this book especially stresses this function of linguistic awareness.

Other mechanisms may have developed in order to prevent failure. In the last section we mentioned the shallow processing which seems involved in 
judging the acceptability or meaningfulness of an utterance. One might guess that such "monitoring for meaningfulness" does not only take place as a consequence of a direct metalinguistic query, but is a general mechanism involved in normal language understanding. It is known that we do monitor speech we hear for specific acoustic features in order to get some "standards" for the correct interpretation of what is to follow. Broadbent and Ladefoged (1960) showed that a listener checks for speaker-specific vowel formants in the first few syllables a new speaker says, and uses these to interpret the vowels to come. It is not likely that a listener makes only phonetic checks. More plausibly he also performs syntactic and semantic ones and these may also lower the probability of comprehension failures. Presented with Chomsky's colorless green ideas sleep furiously the listener probably will not go into an elaborate semantic memory search in order to understand: a shallow preliminary analysis makes it clear that the sentence is meaningless. In this way, superficial checks and pretests may have a monitoring function which ${ }^{-\cdots}$ prevents unnecessary processing and false interpretation.

Apart from facilitating communication in various ways, linguistic awareness may play a functional role in the acquisition of comunicative skills. This was mentioned in passing in the preceding section in connection with the acquisition of skills in general. The point was made there that, to acquire complex skills, a great deal of attention is often required in order to correctly perform the elementary actions of the complex action pattern. Fluent integration of the skill only follows the automatization of activities at the elementary level. It is not clear, and certainly not obvious, that this is true for language acquisition as well. But if it is, one would expect young, i.e., less skilled, language users to be more aware than mature speakers of some of the details involved in speaking. The younger child might need to work consciously on superficial aspects of its language, such as morphological and articulatory details, whereas the older child is fluent in these respects and can direct his attention elsewhere. The evidence for this somewhat paradoxical hypothesis--that the young child is more aware of certain properties of language and speech than the older one--is admittedly scarce. Read (this volume) does show that some phonetic judgments deteriorate with age. Adults who are familiar with written forms have to work in order to re-acquire distinctions that kindergarten children can make easily. Zei (1978), on the other hand, found no evidence that five-year olds were better able to explain the articulatory events involved in speaking than nine-year 
olds. In her study, no comparison was made with adults, however. The issue deserves further attention. It may be especially worthwhile to investigate which aspects of early speaking and listening activities do receive explicit attention during acquisition, and which do not. Knowing this might tell us something about the procedures by which the child acquires linguistic skill, and about how speaking and listening are organized at different ages.

This brings us finaliy to the issue of what feedback structure is involved in the acquisition of speech skills. It is essential in the learning of any skill that the learner acquire internal standards in order to evaluate his own performance. Feedback is not only necessary for comparing standard and performance so that deviations which exceed a certain criterion may be corrected, but also for the development of the internal standards themselves. one theoretically-minded approach, developed by Marshall and Morton in their contribution to this volume, is to describe early forms of linguistic awareness in terms of feedback mechanisms which are involved in the acquisition of basic linguistic skills. The child's monitoring of (others' and especially his own) speech may provide him with the opportunity to check and thereby raise his own standards of production. Marshall and Morton put forth the interesting hypothesis that, as the child learns to speak, the perceptual system "teaches" the production system in approximately this way.

These and similar notions of the functions of linguistic awareness in language acquisition may well have some face validity. However, as yet none of them has been tested experimentally. And experimental tests are necessary. Descriptive evidence will not be sufficient, since correlational findings cannot answer the essential question of whether conscious awareness of language does contribute to first language learning, and if so, whether it is a necessary condition as well. Because of the ethics of human subjects research, the latter part of this question will probably never be answered. The former part, however, is certainly with in the reach of systematic experimental study. We hope that this book will contribute to the development of such research.

REFERENCES

Braine, M.D.S., \& Wells, R.S. Case-like categories in children: The actor and some related categories. Cognitive Psychology, 1978, 10, 100-122.

Broadbent, D.E., \& Ladefoged, P. Vowel judgments and adaptation level. Proceedings of the Royal Society, 1960, 151, 384-399. 
Bühler, K. Tatsachen und Probleme zu einer Psychologie der Denkvorgänge. Archiv für die Gesamte Psychologie, I. Über Gedanken, 1907, 9, 297-305; II . über Gedankenzusammenhänge, 1908, 12, 1-23; II I. Über Gedankenerinnerungen, 1908, 12, 24-92.

De Groot, A.K. Thought and choice in chess. The Hague: Mouton, 1965.

Foppa, K. Language acquisition--a human ethological problem? Social Science Information, 1978, 17, 93-105.

Gleitman, L.R., Gleitman, H., \& Shipley, E.F. The emergence of the child as grammarian. Cognition, 1972, 1, 137-164.

Kaper, W. Kindersprachforschung mit Hilfe des Kindes: Einige Erscheinungen der kindlichen Spracherwerbung erläutert im Lichte des vom Kinde gezeigten Interessen für Sprachliches. Groningen: Wolters, 1959.

Levelt, W.J.M. Some psychological aspects of linguistic data. Linguistische Berichte, 1972, 17, 18-30.

Levelt, W.J.M. Formal gramars in linguistics and psycholinguistics. Vol. III Psycholinguistic applications. The Hague: Mouton, 1974.

Levelt, W.J.M. Skill theory and language teaching. Fourth Colloquium in Theoretical Models in Applied Linguistics. To appear in Studies in second Zanguage acquisition. Bloomington: Indiana University Linguistics Club, 1978.

Levelt, W.J.M., Van Gent, J.A.W.M., Haans, A.F.J., \& Meyers, A.J.A. Grammaticality, paraphrase and imagery. In: S. Greenbaum (ed.), Acceptability in Zanguage. The Hague: Mouton, 1977.

Levelt, W.J.M., \& Kempen, G. Language. In: J.A. Michon, E.G.J. Eykman \& L.F.W. de Klerk (eds.), Handbook of Psychonomics. Amsterdam: Elsevier, 1978, in press.

Mistler-Lachman, J.L. Levels of comprehension of normal and ambiguous sentences. Journal of Verbal Learning and Verbal Behavior, 1972, 11, 614623.

Newell, A., \& Simon, H.A. Human problem solving. Englewood Cliffs: Prentice $\mathrm{Ha} 11,1972$.

Nisbett, R.E., \& Decamp Wilson, T. Telling more than we know: verbal reports on mental processes. Psychological Review, 1977, 84, 231-259.

Papandropoulou, I., \& Sinclair, H. What is a word? Experimental study of children's ideas on grammar. Human Development, 1974, 17, 241-258.

Piaget, J. La prise de conscience. Paris: Presses Universitaires de France, 1974(a).

Piaget, J. Réussir et comprendre. Paris: Presses Universitaires de France, 1974 (b) .

Robinson, E.J., \& Robinson, W.P. Development in the understanding of causes of success and failure in verbal communication. Cognition, 1977, 5, 363-378.

Schegloff, E.A., Jefferson, G., \& Sacks, H. The preference for self-correction in the organization of repair in conversation. Language, 1977, $53,361-382$.

Weir, R.H. Language in the crib. The Hague: Mouton, 1962.

Zei, B. Psychological reality of phonemes. Child Language, 1978, in press. 